\title{
Subtração de números inteiros na reta geométrica
}

\author{
Silvio Esquinca
}

\section{Resumo}

O uso da linguagem geométrica, para o ensino de subtração de números inteiros, em particular na reta de números inteiros, ensinada aos sétimos anos do ensino fundamental, pode trazer uma melhor aprendizagem desse conteúdo ao aluno, de forma que possa, posteriormente, compreender as regras de sinais como um método prático para se obter agilidade com os cálculos.

Palavras-chave: Subtração; Números; Inteiros; Geometria; Reta.

\section{Abstract}

The use of geometric language, for the teaching of subtraction of integer numbers, in particular in the line of integer nembers, taught in the seventh year of elementary school, can bring a better learning of this content to the student, so that he can later understand the signal rules as a practical method for agility with calculations.

Keywords: Subtraction; Numbers; Integers; Geometry; Line

\section{Introdução}

No segundo dia de formação, promovido pela Semed, Secretaria Municipal de Educação, de Campo Grande-MS, cujo tema foi "Organização do Trabalho Didático", para professores da rede municipal de ensino, sendo a Escola Municipal Professor Plínio Mendes dos Santos um polo para o encontro de professores, em março de 2018, levantou-se a questão de como ensinar subtração de números inteiros, geometricamente na reta de números inteiros, para tornar mais acessível este conteúdo ao aluno. Assim, foi apresentada uma abordagem desse conteúdo no livro: Vontade de Saber, $7^{\circ}$ ano, página 105, a qual segue na figura abaixo:

Mas nessa abordagem, o aluno deve saber que $-(-)$ equivale a $+(+)$ e gostaríamos de apresentar um método prático, de melhor compreensão, sem a necessidade do uso dessa equivalência, para depois a compreendermos.

\section{Desenvolvimento}

Os profissionais que ministravam a formação, buscaram uma outra alternativa para abordar a expressão $(-17)-(-28)$ de modo a obter o seu valor 11 , geometricamente na reta de números inteiros, apresentando a figura abaixo: 


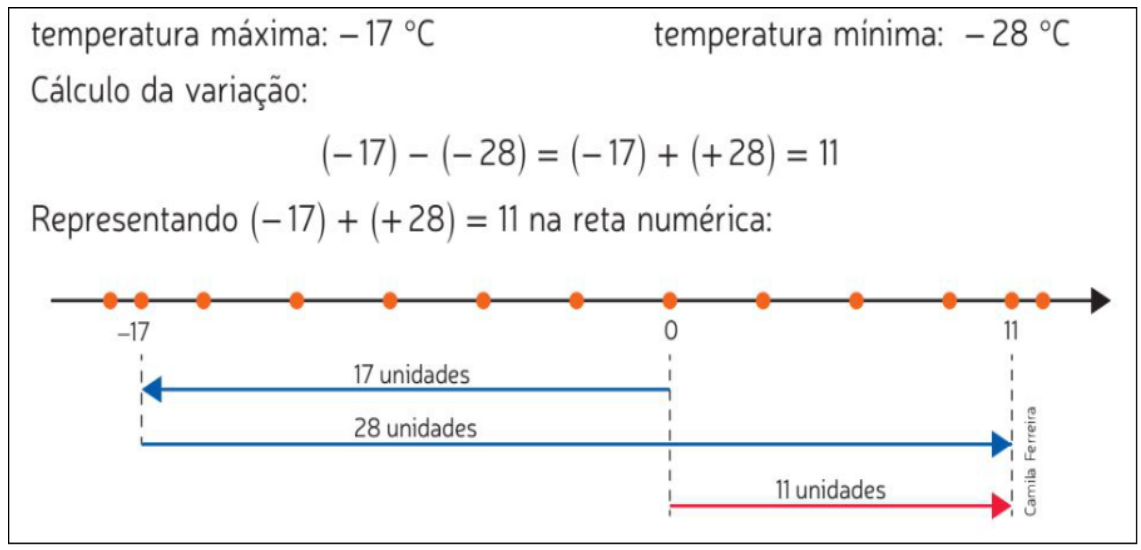

Figura 1: Subtração de números inteiros na reta geométrica de números inteiros.

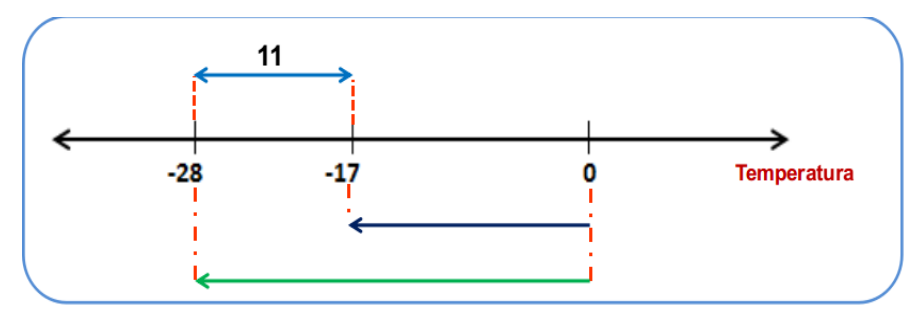

Figura 2: Subtração de números inteiros na reta geométrica de números inteiros.

E com relação a esta abordagem, foram feitas algumas explicações para se tentar chegar no valor 11 para a expressão $(-17)-(-28)$, objetivando encontrar uma praticidade nos esquemas montados na figura.

Algumas reflexões sobre o conteúdo: Subtração de Números Inteiros, contextualizado com a geometria, em particular com a reta de números inteiros, permitiram obter o raciocínio descrito no texto abaixo.

Sejam x e y dois números inteiros quaisquer. É verdade, já demonstrada em livros de vários autores de matemática, que:

$$
|\mathrm{x}-\mathrm{y}|=|\mathrm{y}-\mathrm{x}|
$$

significando: "a distância, na reta, do ponto associado ao número inteiro x ao ponto associado ao número inteiro $\mathrm{y}$, independentemente do sentido, se do ponto associado a $\mathrm{x}$ para o ponto associado a y ou do ponto associado a y para o ponto associado a x. Simplesmente, o módulo fornece apenas tal distância.

Acontece que, trabalhando sem o módulo, a expressão x - y também significará geometricamente, na reta, a distância entre os pontos associados a x e y, porém sendo necessária a indicação de um sentido, do ponto associado a x para o ponto associado a y ou do ponto associado a y para o ponto associado a $\mathrm{x}$.

A leitura da expressão x - y é feita da esquerda para a direita, permitindo extrair, geome- 
tricamente, a seguinte interrogação: "o ponto associado a x distancia-se do ponto associado a y para qual sentido e em quantas unidades de distância na reta?"

A reta possui dois sentidos, um representado pelo sinal + e outro pelo sinal - , conforme figura abaixo.

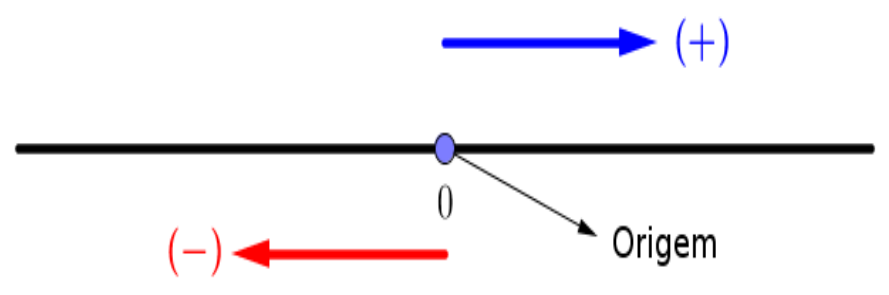

Figura 3: Sentidos na reta.

Se $\mathrm{x}=\mathrm{y}$ então não haverá distanciamento entre os pontos associados a $\mathrm{x}$ e $\mathrm{y}$, consequentemente não existirá sentido, pois os dois pontos serão congruentes. Caso $\mathrm{x}<\mathrm{y}$, haverá uma distância entre os pontos da reta associados a eles, o ponto associado a x permanecendo à esquerda do ponto associado a y. Portanto, o sentido de distanciamento do ponto associado a x, com relação ao ponto associado a y, será indicado pelo sinal de menos (-), conforme a figura abaixo.

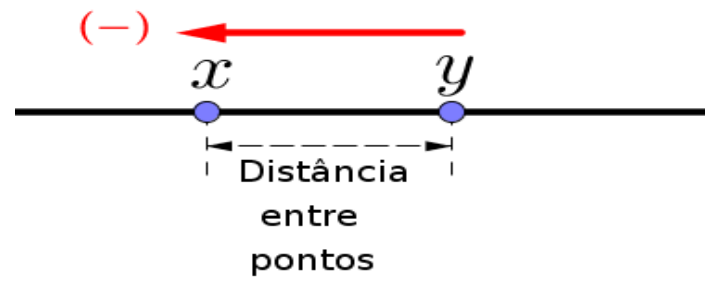

Figura 4: Sentido de um ponto com relação a outro ponto dado.

Mas se $\mathrm{x}>\mathrm{y}$, também haverá uma distância entre os pontos associados a eles, o ponto associado a x permanecendo à direita do ponto associado a y. Portanto, o sentido de distanciamento do ponto associado a x, com relação ao ponto associado a y, será indicado pelo sinal de mais $(+)$, conforme a figura abaixo.

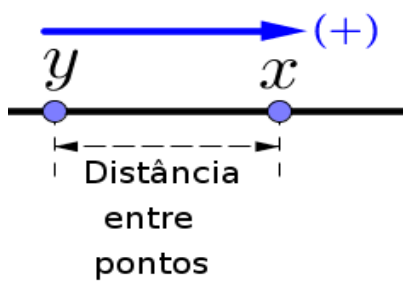

Figura 5: Sentido de um ponto com relação a outro ponto dado. 


\section{Conclusão}

Assim, podemos fornecer os seguintes exemplos com interpretação geométrica:

a)

\begin{tabular}{|c|c|c|c|c|c|}
\hline$(-5)$ & - & $(-3)$ & $=$ & - & 2 \\
\hline $\begin{array}{c}\text { O ponto } \\
\text { associado } \\
\text { ao (-5) }\end{array}$ & $\begin{array}{c}\text { distancia- } \\
\text { se }\end{array}$ & $\begin{array}{c}\text { do ponto } \\
\text { associado } \\
\text { ao (-3) }\end{array}$ & no & $\begin{array}{c}\text { sentido } \\
\text { negativo }\end{array}$ & $\begin{array}{c}\text { duas } \\
\text { unidades } \\
\text { de } \\
\text { distância }\end{array}$ \\
\hline
\end{tabular}

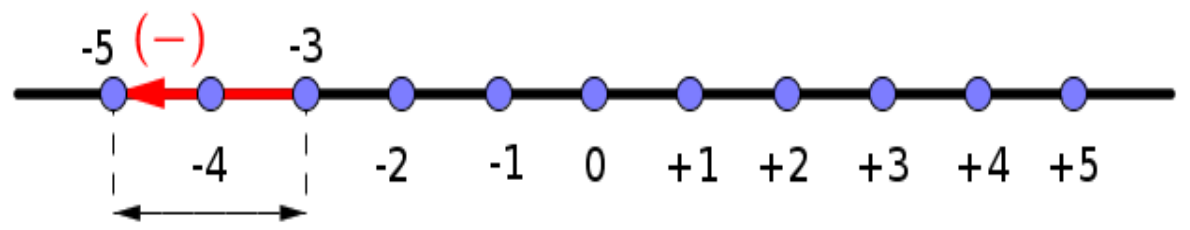

\section{2 unidades}

\section{de distância}

Figura 6: (-5) - (-3) na reta geométrica.

Observação 1: na reta de números inteiros, -5 posiciona-se à esquerda de -3 . Então, como a contagem de unidades de distância ocorrem a partir do -3 , para a esquerda, até chegar em -5 , adota-se o sinal de menos (-) seguido de duas unidades de distância, para o resultado procurado, ou seja, obtendo-se o valor -2 para tal expressão numérica.

b)

\begin{tabular}{|c|c|c|c|c|c|}
\hline$(-5)$ & - & $(+3)$ & $=$ & - & 8 \\
\hline $\begin{array}{c}\text { O ponto } \\
\text { associado } \\
\text { ao (-5) }\end{array}$ & $\begin{array}{c}\text { distancia- } \\
\text { se }\end{array}$ & $\begin{array}{c}\text { do ponto } \\
\text { associado } \\
\text { ao }(+3)\end{array}$ & no & $\begin{array}{c}\text { sentido } \\
\text { negativo }\end{array}$ & $\begin{array}{c}\text { oito } \\
\text { unidades } \\
\text { de } \\
\text { distância }\end{array}$ \\
\hline
\end{tabular}

equivalente a

\begin{tabular}{|c|c|c|c|c|c|}
\hline$(-5)$ & - & 3 & $=$ & - & 8 \\
\hline $\begin{array}{c}\text { O ponto } \\
\text { associado } \\
\text { ao (-5) }\end{array}$ & $\begin{array}{c}\text { distancia- } \\
\text { se }\end{array}$ & $\begin{array}{c}\text { do ponto } \\
\text { associado } \\
\text { ao } 3=+3\end{array}$ & no & $\begin{array}{c}\text { sentido } \\
\text { negativo }\end{array}$ & $\begin{array}{c}\text { oito } \\
\text { unidades } \\
\text { de } \\
\text { distância }\end{array}$ \\
\hline
\end{tabular}




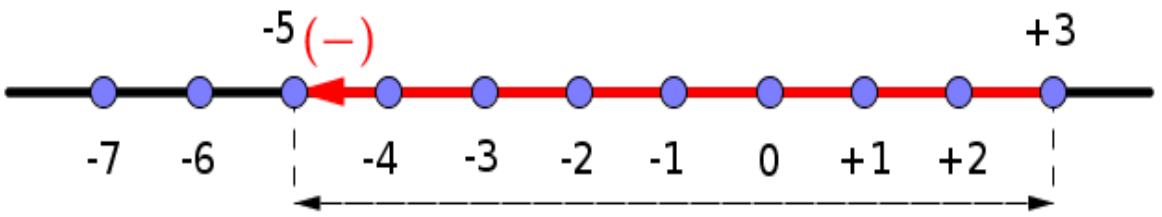

8 unidades de distância

Figura 7: $(-5)-(+3)$ na reta geométrica.

c)

\begin{tabular}{|c|c|c|c|c|c|}
\hline$(-3)$ & - & $(-5)$ & $=$ & + & 2 \\
\hline $\begin{array}{c}\text { O ponto } \\
\text { associado } \\
\text { ao (-3) }\end{array}$ & $\begin{array}{c}\text { distancia- } \\
\text { se }\end{array}$ & $\begin{array}{c}\text { do ponto } \\
\text { associado } \\
\text { ao (-5) }\end{array}$ & no & $\begin{array}{c}\text { sentido } \\
\text { positivo }\end{array}$ & $\begin{array}{c}\text { duas } \\
\text { unidades } \\
\text { de } \\
\text { distância }\end{array}$ \\
\hline
\end{tabular}

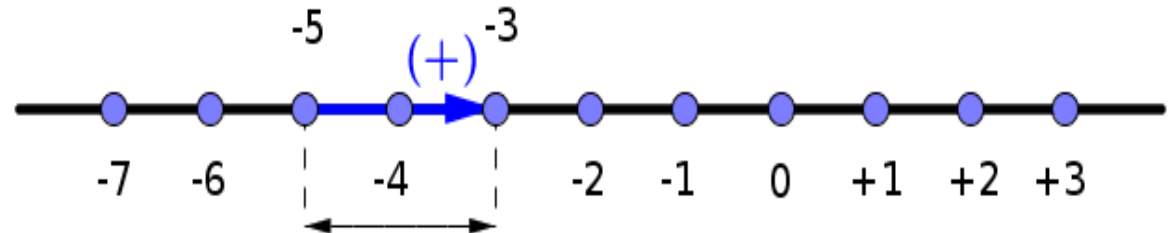

2 unidades

de distância

Figura 8: (-3) - (-5) na reta geométrica.

Observação 2: na reta de números inteiros, -3 posiciona-se à direita de -5 . Então, como a contagem de unidades de distância ocorrem a partir do -5 , para a direita, até chegar em -3, adota-se o sinal de mais $(+)$ seguido de duas unidades de distância, para o resultado procurado, ou seja, obtendo-se o valor $+2=2$ para tal expressão numérica.

d)

\begin{tabular}{|c|c|c|c|c|c|}
\hline$(-3)$ & - & $(+5)$ & $=$ & - & 8 \\
\hline $\begin{array}{c}\text { O ponto } \\
\text { associado } \\
\text { ao (-3) }\end{array}$ & $\begin{array}{c}\text { distancia- } \\
\text { se }\end{array}$ & $\begin{array}{c}\text { do ponto } \\
\text { associado } \\
\text { ao }(+5)\end{array}$ & no & $\begin{array}{c}\text { sentido } \\
\text { negativo }\end{array}$ & $\begin{array}{c}\text { oito } \\
\text { unidades } \\
\text { de } \\
\text { distância }\end{array}$ \\
\hline
\end{tabular}

equivalente a 


\begin{tabular}{|c|c|c|c|c|c|}
\hline$(-3)$ & - & 5 & $=$ & - & 8 \\
\hline $\begin{array}{c}\text { O ponto } \\
\text { associado } \\
\text { ao (-3) }\end{array}$ & distancia- & $\begin{array}{c}\text { do ponto } \\
\text { associado } \\
\text { ao } 5=+5\end{array}$ & no & $\begin{array}{c}\text { sentido } \\
\text { negativo }\end{array}$ & $\begin{array}{c}\text { oito } \\
\text { unidades } \\
\text { de } \\
\text { distância }\end{array}$ \\
\hline
\end{tabular}

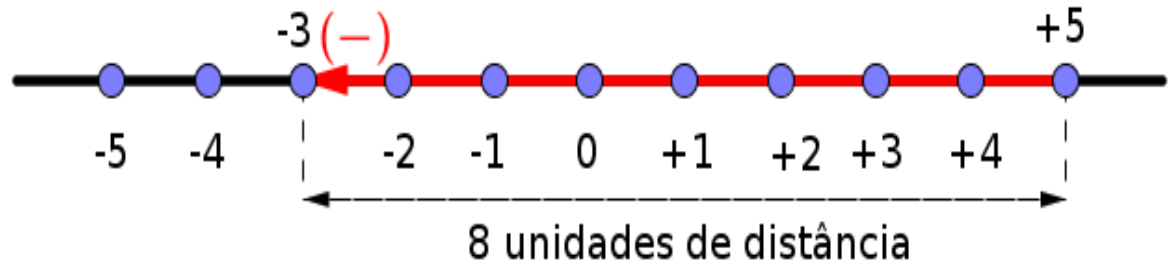

Figura 9: $(-3)-(+5)$ na reta geométrica.

e)

\begin{tabular}{|c|c|c|c|c|c|}
\hline$(+5)$ & - & $(+3)$ & $=$ & + & 2 \\
\hline $\begin{array}{c}\text { O ponto } \\
\text { associado } \\
\text { ao }(+5)\end{array}$ & $\begin{array}{c}\text { distancia- } \\
\text { se }\end{array}$ & $\begin{array}{c}\text { do ponto } \\
\text { associado } \\
\text { ao }(+3)\end{array}$ & no & $\begin{array}{c}\text { sentido } \\
\text { positivo }\end{array}$ & $\begin{array}{c}\text { duas } \\
\text { unidades } \\
\text { de } \\
\text { distância }\end{array}$ \\
\hline
\end{tabular}

equivalente a

\begin{tabular}{|c|c|c|c|c|c|}
\hline$(+5)$ & - & 3 & $=$ & + & 2 \\
\hline $\begin{array}{c}\text { O ponto } \\
\text { associado } \\
\text { ao }(+5)\end{array}$ & $\begin{array}{c}\text { distancia- } \\
\text { se }\end{array}$ & $\begin{array}{c}\text { do ponto } \\
\text { associado } \\
\text { ao } 3=+3\end{array}$ & no & $\begin{array}{c}\text { sentido } \\
\text { positivo }\end{array}$ & $\begin{array}{c}\text { duas } \\
\text { unidades } \\
\text { de } \\
\text { distância }\end{array}$ \\
\hline
\end{tabular}

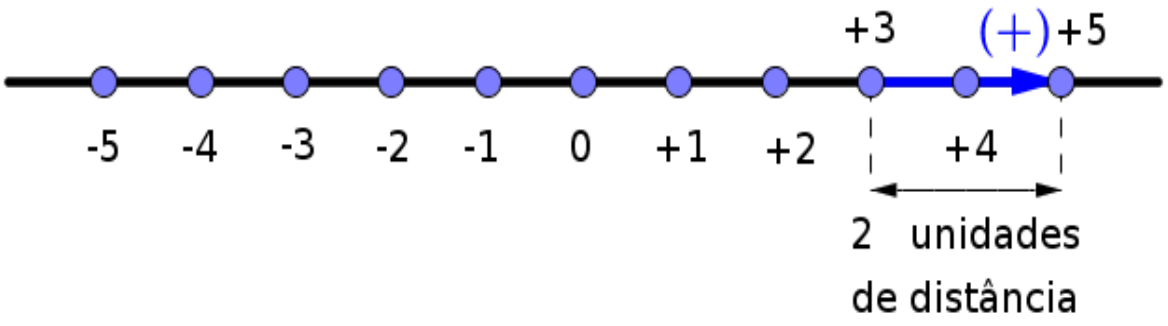

Figura 10: $(+5)-(+3)$ na reta geométrica.

f) 


\begin{tabular}{|c|c|c|c|c|c|}
\hline$(+3)$ & - & $(+5)$ & $=$ & - & 2 \\
\hline $\begin{array}{c}\text { O ponto } \\
\text { associado } \\
\text { ao }(+3)\end{array}$ & distancia- & $\begin{array}{c}\text { do ponto } \\
\text { associado } \\
\text { ao }(+5)\end{array}$ & no & $\begin{array}{c}\text { sentido } \\
\text { negativo }\end{array}$ & $\begin{array}{c}\text { duas } \\
\text { unidades } \\
\text { de } \\
\text { distância }\end{array}$ \\
\hline
\end{tabular}

equivalente a

\begin{tabular}{|c|c|c|c|c|c|}
\hline 3 & - & 5 & $=$ & - & 2 \\
\hline $\begin{array}{c}\text { O ponto } \\
\text { associado } \\
\text { ao } 3=+3\end{array}$ & $\begin{array}{c}\text { distancia- } \\
\text { se }\end{array}$ & $\begin{array}{c}\text { do ponto } \\
\text { associado } \\
\text { ao } 5=+5\end{array}$ & no & $\begin{array}{c}\text { sentido } \\
\text { negativo }\end{array}$ & $\begin{array}{c}\text { duas } \\
\text { unidades } \\
\text { de } \\
\text { distância }\end{array}$ \\
\hline
\end{tabular}

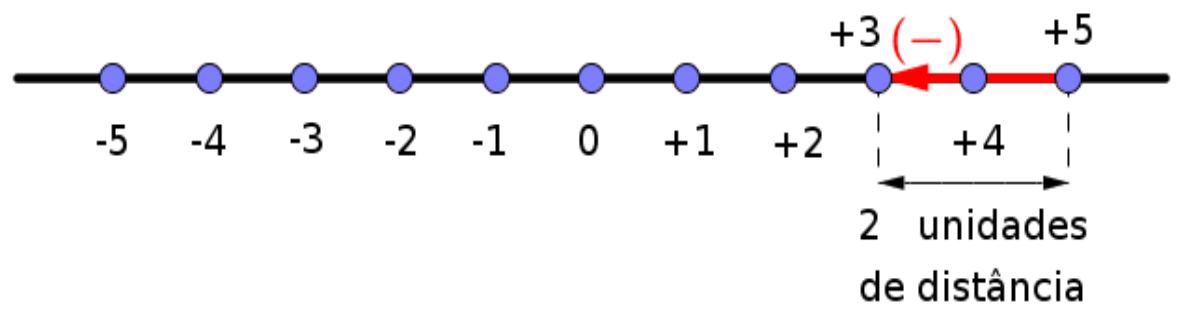

Figura 11: $(+3)-(+5)$ na reta geométrica.

\section{Referências}

[1] SOUZA, Joamir e PATARO, Patrícia Moreno. Vontade de Saber, $7^{\mathrm{o}}$ Ano. São Paulo: FTD, 2015.

[2] LEITHOLD, Louis. Cálculo com Geometria Analítica. Tradução: Cyro de Carvalho Patarra. São Paulo: Harbra, 1994.

Silvio Esquinca

Secretaria Municipal de Educação de Campo Grande-MS

Escola Municipal Prof. Plínio Mendes dos Santos Secretaria de Estado de Educação de Mato Grosso do Sul Escola Estadual José Ferreira Barbosa $<$ profesquinca@gmail.com> 